Original Article

\title{
Comparison of range of motion during movement from supine to sitting position in healthy young and elderly participants
}

Tetsuro Watanabe, PT, MSc ${ }^{1,2)^{*}}$, Hisayoshi Ogihara, PT, PhD ${ }^{3)}$, Takehito Soeta, PT ${ }^{1)}$, Takayuki Fujiwara, PT, DDM, PhD, DE ${ }^{2,4)}$, Hiroaki Yoshida, DE ${ }^{2}$

1) Department of Physical Therapy, Koriyama Institute of Health Sciences: 2-9-3 Zukei, Koriyama city, Fukushima 963-8834, Japan

2) Interdisciplinary Graduate School of Science and Technology, Shinshu University, Japan

3) Regional Cooperation Center, Iwaki Meisei University, Japan

4) Graduate School of Science and Technology, Iwaki Meisei University, Japan

\begin{abstract}
Purpose] The purpose of this study was to compare the range of motion (ROM) during supine-tositting position (StSP) movement between healthy young and elderly participants to understand age-related tendencies, which is beneficial for the assessment and treatment of frail elderly. [Participants and Methods] The participants were 14 healthy young males (age, 19-24 years) and 16 healthy elderly individuals ( 8 males and 8 females; age, 65-74 years). Rising movement was performed 5 times freely at a comfortable speed. [Results] The joint angles during StSP movements in right shoulder abduction, extension of both shoulder joints, right elbow flexion, trunk extension, and adduction of both hip joints were significantly larger, whereas neck flexion, trunk flexion, and left hip joint abduction were significantly smaller in the healthy elderly participants than in the healthy young participants. All joint movements were earlier in the elderly participants than in the healthy young participants. [Conclusion] The results of this study suggest that focusing on how to use both upper limbs is important, in addition to the neck and trunk, when evaluating StSP movement. Similarly, to encourage independence during StSP movement, the elbow extensors must be strengthened.

Key words: Rising movement, Quantitative analysis, Three-dimensional motion analysis systems
\end{abstract}

(This article was submitted Aug. 30, 2018, and was accepted Oct. 24, 2018)

\section{INTRODUCTION}

Moving from supine to sitting position (StSP) is important for activities of daily living. Previous studies investigating StSP movement have largely been qualitative and currently no quantitative analysis of this type of movement could be found. Most of the qualitative studies reported their methodology as using digital video cameras, capturing movement from the perspective of human development ${ }^{1-5}$. Many of these studies used classification tables that were divided into four body regions; head and trunk, distal arm, proximal arm, and legs. Many of the studies on the variability of StSP movement, and none reported on the relationship of the movement to physical function. Additionally, with regards to stroke patients and the community-dwelling elderly, in some studies the StSP movement is considered using quantitative parameters such as duration and functional index ${ }^{6-8)}$. Additionally, one study investigated the getting up movement from the floor ${ }^{9}$.

As StSP movement is performed by synergistic movement of each body segment, quantitative evaluations are more useful than qualitative evaluations. A comparison of range of motion (ROM) during StSP movement in healthy young and healthy elderly participants allows us to understand the age-related tendencies, which is beneficial for the assessment and treatment

*Corresponding author. Tetsuro Watanabe (E-mail: t-watanabe@k-tohto.ac.jp)

(C2019 The Society of Physical Therapy Science. Published by IPEC Inc.

(c) (1) $($ This is an open-access article distributed under the terms of the Creative Commons Attribution Non-Commercial No Derivatives CC. ${ }_{\text {BY }}$ ND (by-nc-nd) License. (CC-BY-NC-ND 4.0: https://creativecommons.org/licenses/by-nc-nd/4.0/) 
of the frail elderly. Therefore, the purpose of this study was to compare ROM during StSP movement in healthy young and healthy elderly participants.

\section{PARTICIPANTS AND METHODS}

The participants were 14 healthy young males (mean age 20 years, range 19 to 24 years) and 16 healthy elderly participants ( 8 males and 8 females, mean age 68 years, range 65 to 74 years). Table 1 is a summary of the participants' profiles. Individuals who reported any orthopedic or neurological conditions (e.g., pain) that would interfere with StSP movement were excluded from the study. The research was conducted in compliance with the Helsinki Declaration and after obtaining the approval of the school corporation from Koriyama Tohto Academy Ethics Committee (approval number: R1502). In addition, all participants received a thorough briefing both verbally and in writing about the potential risk of injury, voluntary participation in the study, and protection of personal information.

Infra-red reflective markers were attached to the participants at 26 body part locations (parietal, left and right temporal regions, forehead, sternal angle, body of sternum, left and right acromion, lateral elbow joint, dorsal middle point of wrist joint, iliac crest, anterior superior iliac spine (ASIS), lateral knee joint, front of ankle joint, third metatarsal) (Fig. 1). Marker information during StSP movement was measured using a three dimensional motion analysis system (Mac 3D system, Motion Analysis Co.) using nine infra-red cameras. The sampling frequency (frame rate) was set at $100 \mathrm{~Hz}$. Angle and time information were extracted using a data integration analysis program (KineAnalyzer, KISSEI COMTEC Co.). The StSP movement was performed on a platform (length $120 \mathrm{~cm}$, width $120 \mathrm{~cm}$, and height $41 \mathrm{~cm}$ ).

In order to standardize the starting position, participants were asked to keep their upper limbs by the side of the body with the palmar surface of the hand resting on the platform. The StSP movement started from the supine position on the platform with an oral cue ("Ready. Go"), spoken from the right side of the platform, and participants were instructed to reply "Yes" at the time when they felt that the movement was over (Fig. 2). Practice exercises of StSP movement were performed until the participants themselves felt that they had practiced sufficiently. The StSP movement was performed 5 times freely at a comfortable speed.

A Butterworth filter processing was performed at $6 \mathrm{~Hz}$ for noise cancellation of the marker locus. The analysis of the movement was from the oral cue until the participant replied. Data was normalized based on samples of the shortest data among healthy young and healthy elderly participants. The average value of the angle of the five StSP movements, and the

Table 1. Participants profiles

\begin{tabular}{lcc}
\hline & Young & Elderly \\
\hline Mean age (range) (years) & $20(19-24)$ & $69(65-74)$ \\
Height $(\mathrm{cm})$ & $168.8 \pm 6.2$ & $157.7 \pm 7.4$ \\
Body weight $(\mathrm{kg})$ & $62.9 \pm 4.9$ & $50.8 \pm 9.5$ \\
BMI $\left(\mathrm{kg} / \mathrm{m}^{2}\right)$ & $22.2 \pm 2.2$ & $20.4 \pm 3.1$ \\
\hline
\end{tabular}

Mean \pm SD.

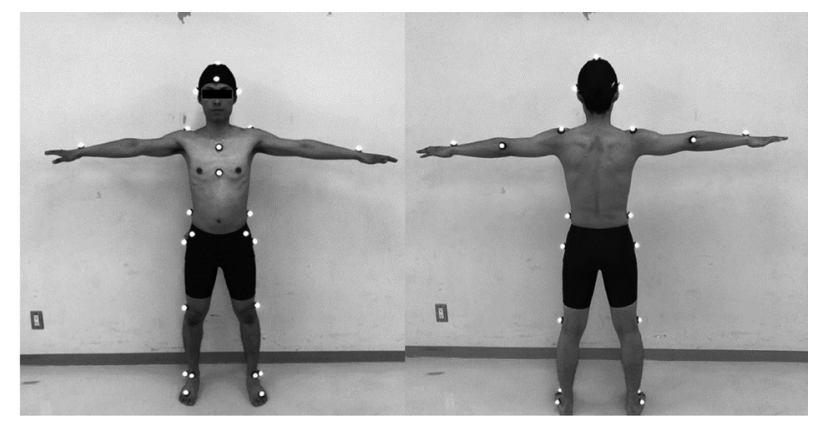

Fig. 1. Infra-red reflective marker pasting position.

Infra-red reflective markers were pasted to 26 participants' body parts (Parietal, left and right temporal regions, forehead, sternal angle, body of sternum, left and right acromion, lateral elbow joint, dorsal middle point of wrist joint, iliac crest, anterior superior iliac spine (ASIS), lateral knee joint, front of ankle joint, third metatarsal).

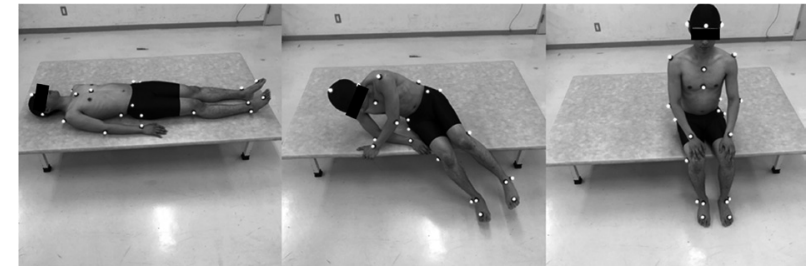

Fig. 2. Rising movement.

In order to standardize the starting position, participants were asked to keep their upper limbs by the side of the body with the palmar surface of the hand resting on the platform. The StSP movement started from the supine position on the platform with an oral cue ("Ready. Go"), spoken from the right side of the platform, and participants were instructed to reply "Yes" at the time when they felt that the movement was over. 


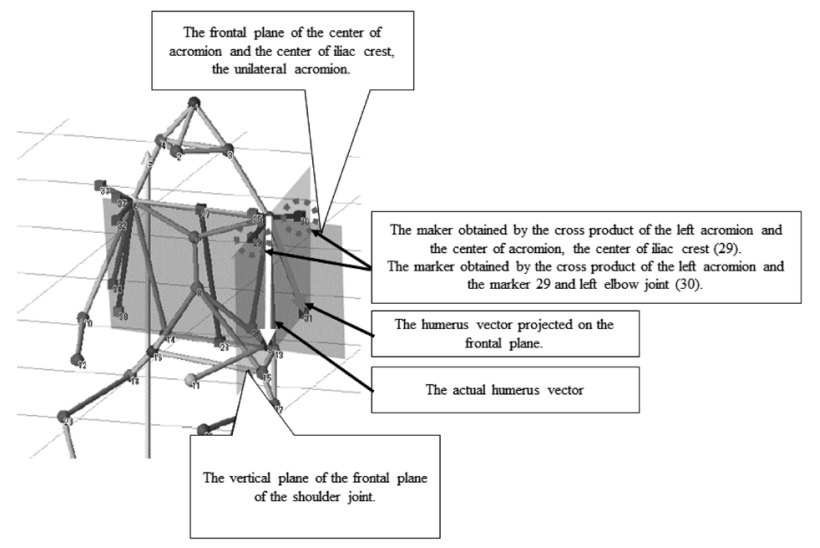

Fig. 3. The example of the joint angle definition (shoulder joint adduction/ abduction).

Define the frontal plane from 3 arbitrary markers. After extract the cross product from the movement plane, extract the humerus vector and the new cross product vector which was the movement axis. The angle formed by the humerus vector projected on the movement plane and the basic axis was defined as the joint angle in this study.

Table 2. The median and quartile of the angle

\begin{tabular}{|c|c|c|c|c|c|c|}
\hline & \multicolumn{3}{|c|}{ Young } & \multicolumn{3}{|c|}{ Erderly } \\
\hline & Median $\left({ }^{\circ}\right)$ & First quartile & Third quartile & Median $\left(^{\circ}\right)$ & First quartile & Third quartile \\
\hline Right shoulder adduction & 13.9 & 10.0 & 15.7 & 9.4 & 6.9 & 11.7 \\
\hline Right shoulder abduction* & 28.8 & 25.9 & 44.3 & 42.7 & 35.6 & 51.2 \\
\hline Left shoulder adduction** & 12.2 & 8.7 & 14.2 & 5.0 & -1.5 & 10.3 \\
\hline Left shoulder abduction & 24.8 & 19.4 & 31.3 & 27.7 & 22.2 & 31.6 \\
\hline Right shoulder flexion & 25.9 & 17.9 & 34.2 & 27.7 & 20.3 & 30.8 \\
\hline Right shoulder extension** & -21.9 & -24.6 & -17.4 & -31.3 & -38.2 & -27.3 \\
\hline Left shoulder flexion & 20.2 & 16.5 & 27.9 & 20.9 & 9.1 & 25.9 \\
\hline Left shoulder extension** & -17.2 & -21.0 & -13.3 & -32.9 & -39.5 & -27.1 \\
\hline Right elbow flexion** & 84.1 & 71.7 & 89.2 & 92.2 & 80.2 & 96.1 \\
\hline Right elbow extension & 25.7 & 21.9 & 30.1 & 24.0 & 20.7 & 28.3 \\
\hline Left elbow flexion & 64.6 & 52.0 & 75.6 & 67.5 & 59.5 & 78.8 \\
\hline Left elbow extension & 25.5 & 19.6 & 31.7 & 23.1 & 17.5 & 24.7 \\
\hline Neck right rotation & 22.1 & 12.2 & 25.2 & 19.7 & 11.6 & 25.0 \\
\hline Neck left rotation & -3.8 & -6.5 & -0.6 & -6.7 & -14.4 & -2.1 \\
\hline Neck flexion & 34.6 & 21.5 & 43.2 & 38.3 & 30.4 & 45.0 \\
\hline Neck extension** & -27.5 & -36.2 & -18.8 & -8.5 & -15.8 & -3.2 \\
\hline Trunk right rotation & 5.3 & 1.5 & 10.4 & 5.9 & 2.6 & 12.0 \\
\hline Trunk left rotation & -14.2 & -17.5 & -9.0 & -14.3 & -19.8 & -10.2 \\
\hline Trunk flexion** & 63.2 & 46.9 & 68.6 & 37.2 & 30.5 & 40.7 \\
\hline Trunk extension** & 2.4 & 0.2 & 10.8 & -15.5 & -19.1 & -9.8 \\
\hline Right hip joint adduction** & -5.8 & -7.2 & -3.2 & -12.0 & -15.2 & -9.7 \\
\hline Right hip joint abduction & 21.0 & 14.0 & 28.2 & 11.2 & 4.5 & 24.3 \\
\hline Left hip joint adduction* & -13.8 & -15.3 & -9.9 & -17.7 & -22.8 & -12.9 \\
\hline Left hip joint abduction* & 3.3 & 1.6 & 8.8 & -2.1 & -4.1 & 5.0 \\
\hline Right hip joint flexion** & 56.2 & 45.9 & 63.0 & 80.0 & 74.4 & 91.2 \\
\hline Right hip joint extension** & 9.9 & 8.0 & 14.6 & 25.3 & 18.1 & 27.6 \\
\hline Left hip joint flexion & 64.0 & 45.0 & 73.9 & 71.6 & 66.4 & 86.5 \\
\hline Left hip joint extension & 7.2 & 4.6 & 12.1 & 13.2 & 9.2 & 20.9 \\
\hline Right knee joint flexion** & 55.3 & 47.1 & 64.9 & 77.8 & 69.2 & 86.9 \\
\hline Right knee joint extension** & 10.5 & 9.1 & 11.1 & 14.5 & 11.4 & 17.0 \\
\hline Left knee joint flexion** & 59.7 & 46.6 & 71.5 & 74.1 & 66.0 & 78.3 \\
\hline Left knee joint extension & 10.5 & 6.1 & 11.3 & 11.5 & 9.7 & 13.8 \\
\hline
\end{tabular}

$* \mathrm{p}<0.05, * * \mathrm{p}<0.01$ 
Table 3. The arrival timing of the angle (ATA)

\begin{tabular}{|c|c|c|c|c|c|c|}
\hline & \multicolumn{3}{|c|}{ Young } & \multicolumn{3}{|c|}{ Erderly } \\
\hline & Median (\%) & First quartile & Third quartile & Median (\%) & First quartile & Third quartile \\
\hline Right shoulder adduction & 55.8 & 29.7 & 89.4 & 76.8 & 43.4 & 87.8 \\
\hline Right shoulder abduction* & 51.6 & 44.6 & 63.6 & 41.4 & 33.9 & 50.1 \\
\hline Left shoulder adduction* & 73.8 & 59.4 & 92.6 & 62.5 & 48.5 & 69.6 \\
\hline Left shoulder abduction** & 44.8 & 34.8 & 60.2 & 24.4 & 17.2 & 32.8 \\
\hline Right shoulder flexion** & 79.8 & 77.5 & 87.9 & 69.4 & 60.6 & 77.0 \\
\hline Right shoulder extension & 25.3 & 18.7 & 30.1 & 23.9 & 19.4 & 28.4 \\
\hline Left shoulder flexion & 84.2 & 55.4 & 91.9 & 89.2 & 71.7 & 95.8 \\
\hline Left shoulder extension & 21.4 & 13.1 & 31.5 & 23.7 & 19.8 & 31.6 \\
\hline Right elbow flexion & 41.6 & 31.4 & 46.7 & 34.3 & 24.3 & 43.1 \\
\hline Right elbow extension & 6.3 & 0.9 & 19.8 & 10.4 & 4.3 & 37.1 \\
\hline Left elbow flexion* & 73.5 & 38.5 & 84.2 & 30.6 & 21.9 & 65.9 \\
\hline Left elbow extension & 21.9 & 5.3 & 39.0 & 29.2 & 2.4 & 44.3 \\
\hline Neck right rotation* & 49.7 & 42.9 & 58.1 & 35.6 & 26.9 & 43.9 \\
\hline Neck left rotation** & 24.1 & 18.9 & 41.4 & 53.6 & 36.6 & 63.4 \\
\hline Neck flexion & 27.6 & 22.9 & 33.2 & 26.0 & 22.1 & 37.2 \\
\hline Neck extension** & 87.2 & 73.7 & 94.2 & 58.8 & 6.1 & 78.1 \\
\hline Trunk right rotation & 39.5 & 28.3 & 65.4 & 33.7 & 26.3 & 45.9 \\
\hline Trunk left rotation* & 56.9 & 45.1 & 74.7 & 45.3 & 36.3 & 54.4 \\
\hline Trunk flexion & 53.6 & 47.6 & 60.0 & 53.9 & 51.2 & 64.3 \\
\hline Trunk extension & 0.8 & 0.2 & 2.8 & 0.7 & 0.5 & 3.4 \\
\hline Right hip joint adduction & 70.6 & 39.8 & 75.6 & 57.3 & 37.7 & 63.5 \\
\hline Right hip joint abduction** & 52.4 & 41.4 & 57.8 & 37.1 & 30.1 & 45.7 \\
\hline Left hip joint adduction** & 62.0 & 53.3 & 76.7 & 41.2 & 32.4 & 47.0 \\
\hline Left hip joint abduction & 51.2 & 29.3 & 62.3 & 41.4 & 30.4 & 50.8 \\
\hline Right hip joint flexion & 69.1 & 62.7 & 89.9 & 72.2 & 60.9 & 79.5 \\
\hline Right hip joint extension** & 17.7 & 6.8 & 26.7 & 5.8 & 3.7 & 9.8 \\
\hline Left hip joint flexion* & 84.1 & 78.9 & 89.4 & 69.2 & 59.2 & 74.1 \\
\hline Left hip joint extension* & 25.2 & 19.6 & 30.1 & 9.0 & 3.3 & 16.3 \\
\hline Right knee joint flexion* & 84.9 & 76.2 & 91.3 & 67.3 & 51.3 & 87.5 \\
\hline Right knee joint extension & 6.0 & 0.2 & 7.4 & 2.1 & 0.2 & 3.7 \\
\hline Left knee joint flexion* & 91.8 & 80.7 & 95.6 & 82.3 & 73.1 & 86.5 \\
\hline Left knee joint extension & 6.1 & 1.8 & 15.8 & 1.7 & 0.8 & 4.1 \\
\hline
\end{tabular}

$* \mathrm{p}<0.05, * * \mathrm{p}<0.01$

arrival timing of the angle (ATA) were taken as the representative value. Joint angles were extracted for neck right rotation, flexion/ extension, trunk right rotation, flexion/ extension, shoulder joint adduction/ abduction, flexion/ extension, elbow joint flexion, hip joint adduction/ abduction, flexion/ extension, knee joint flexion.

For statistical analysis the Mann-Whitney's U test was used to investigate the differences between the angle of neck right rotation, flexion, trunk right rotation, flexion, shoulder joint adduction/ abduction, flexion/ extension, elbow joint flexion, hip joint adduction/ abduction and ATA in the healthy young and the healthy elderly. The level of significance was set at 5\%.

In this study, the starting position of the StSP movement was the supine position and the marker could not be attached to the participants' back. Thus, for this reason, the joint angle of three dimensions was defined by obtaining the cross product of vectors from the markers pasted to each part of the body (Fig. 3). All analyses were performed according to the coordinates of the right-hand.

\section{RESULTS}

The median and quartile of the angle of the StSP movement are shown in the Tables 2 and 3. Similarly, Figs. 4-6 shows changes over time in the angle during StSP movement. Joint angle during StSP movement of right shoulder abduction, both shoulder joint extension, right elbow flexion, trunk extension and both hip joint adduction were significantly larger, and neck flexion, trunk flexion and left hip joint abduction were significantly smaller in the healthy elderly than in the healthy young. Additionally, the healthy elderly's joint movement was earlier than the healthy young for all joint movements. 


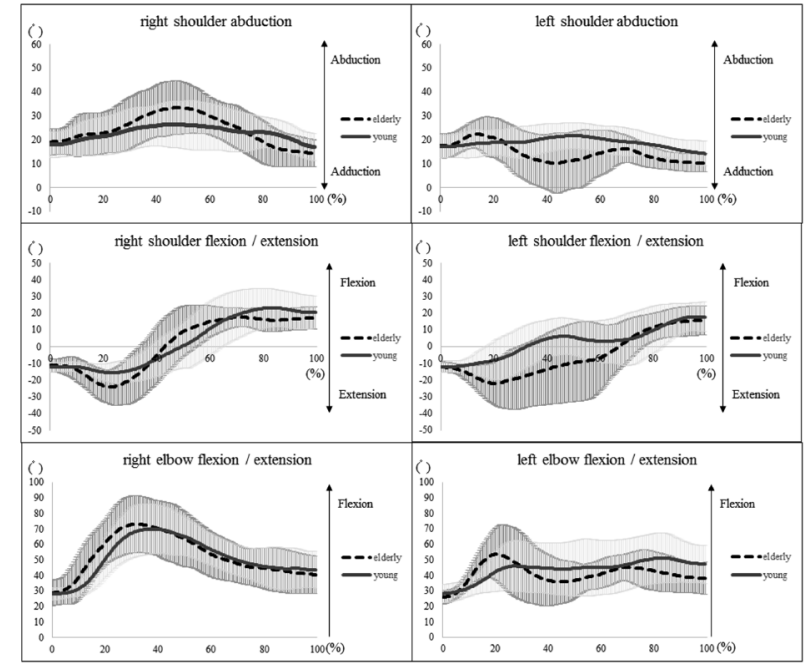

Fig. 4. Changes over time of upper extremity.

\section{DISCUSSION}

This study examined quantitatively the differences in the joint movement during StSP movement in the healthy young and the healthy elderly using a three dimensional motion analysis system. From the ROM results, the joint angles of healthy elderly were larger with both shoulder extension and right elbow joint flexion, than the healthy young. When observing the actual movement, the healthy elderly often selected the push up during StSP movement. In general, muscle strength of the whole body and physical functions decrease due to age-related changes. The StSP movement that changes the posture from the supine position to the sitting position moves the center of mass of the upper body upwards against the influence of gravity, so that the trunk flexors serve an important function. Consequently, it becomes difficult to use trunk flexors due to age-related changes, and thus many participants chose to use a push up movement. Therefore, the angle of the far shoulder joint during rising movement in the healthy young was larger than that of the elderly, and in the healthy elderly, the extension angle of both shoulders was larger than that of the healthy young. Ford-Smith reported that rising patterns change with age ${ }^{1)}$. According to the study by Alexander et al. ${ }^{10)}, 62 \%$ of the elderly who lived in a local community were unable to rise from a bed without using their upper limbs. Furthermore, the report referred to the importance of the upper limbs for trunk elevation. In another study by Alexander et al. ${ }^{11)}$, the healthy elderly were able to rise from various initial positions on the floor, only $63 \%$ of the elderly residents in congregate housing were able to rise from the floor, even if there was support. The results of this study demonstrated that the difference in the joint angles between the healthy young and the healthy elderly suggested that the support of upper limbs was important for StSP movement as in previous studies.

Meanwhile, ATA of the neck right rotation and trunk left rotation were significantly earlier for the healthy elderly than the healthy young (Fig. 5). The reason for this seems to be that during the push up, the head and neck rotation preceded the movement of the trunk segment. Moreover, the healthy elderly had abducted the right shoulder joint and the hip joint from an earlier period during the StSP movement. Kaneko et al. ${ }^{6}$ ) indicated the importance of the lateral balance as a factor influencing the StSP movement from the result of the lateral reach test. Also in this study, it can be inferred that the healthy elderly had a strategy to expand the base of support in order to compensate for the lateral balance function.

Until now, it seems that many therapists are paying attention to the movements of the neck and trunk when instructing rehabilitation patients to get up. The results of this study suggest that focusing on how to use both upper limbs is important, 
in addition to the neck and the trunk, when evaluating the StSP movement. Similarly, in order to encourage independence of the StSP movement, it is necessary to strengthen the elbow extensor. The ROM of the lower limbs was larger in the healthy elderly than the healthy young (Fig. 6). Although the previous studies have reported on lower limbs movements, it was a report limited to the observation of symmetrical movement in the sagittal plane only ${ }^{12}$. In addition to the attention to the movements of the upper limbs, we would like to consider the motion of the lower limbs.

In this study, we focused on the joint movement and its timing. As a result, in the healthy young and elderly, there was no difference in the range of joint during StSP movement, but it did suggest that the timing of the movement was different. Therefore, in the case of the StSP movement performed by a healthy participant, it is presumed that the muscular strength and the timing of the muscle contraction, premotor reaction time are more important than flexibility. For future research, in order to investigate the contribution rate of mobility and strength, we would like to further examine the center of the pressure length and movement of the center of gravity.

A limitation of this study is that dedicated software must be used for the angle definition, as used in this study, and thus it may be impossible to accurately compare this study with other similar studies. Although the Euler angles were not calculated, it is possible to understand the kinematics of StSP movement from the angular data obtained in this study.

\section{Conflict of interest}

There are no conflicts of interest to disclose in this article.

\section{REFERENCES}

1) Ford-Smith CD, VanSant AF: Age differences in movement patterns used to rise from a bed in subjects in the third through fifth decades of age. Phys Ther, 1993, 73: 300-309. [Medline] [CrossRef]

2) Mount J, Kresge L, Klaus L, et al.: Movement patterns used by the elderly when getting out of bed. Phys Occup Ther Geriatr, 2006, 24: 27-43. [CrossRef]

3) Mount J, Cianci H, Welman R, et al.: How people with Parkinson's disease get out of bed. Phys Occup Ther Geriatr, 2009, 27: 333-359. [CrossRef]

4) McCoy JO, VanSant AF: Movement patterns of adolescents rising from a bed. Phys Ther, 1993, 73: 182-193. [Medline] [CrossRef]

5) Banswal SK, Kaur J, Nawaria PB: Rising from bed: comparison between movement patterns of stroke and healthy individuals. Int J Health Sci Res, 2015, 5 : 337-346.

6) Kaneko J, Morala D, Kurosawa K, et al.: Relationship between movement patterns and physical fitness elements during rising from the supine to sitting position in community-dwelling elderly persons. J Phys Ther Sci, 2003, 15: 87-91. [CrossRef]

7) Hachiya M, Murata S, Shingou S, et al.: Relationship between getting up ability and physical function of the elderly. Rigakuryoho Kagaku, 2010, 25: 271-274 (In Japanese). [CrossRef]

8) Kuwabara C, Shiba Y, Sakamoto M, et al.: The relationship between the movement patterns of rising from a supine to an erect stance and physical function in healthy children. Adv Physiol Educ, 2013, 3: 92-97. [CrossRef]

9) Bohannon RB, Lusardi MM: Getting up from the floor. Determinants and techniques among healthy older adults. Physiother Theory Pract, 2004, 20: 233-241. [CrossRef]

10) Alexander NB, Grunawalt JC, Carlos S, et al.: Bed mobility task performance in older adults. J Rehabil Res Dev, 2000, 37: 633-638. [Medline]

11) Alexander NB, Ulbrich J, Raheja A, et al.: Rising from the floor in older adults. J Am Geriatr Soc, 1997, 45: 564-569. [Medline] [CrossRef]

12) Cordo PJ, Gurfinkel VS, Smith TC, et al.: The sit-up: complex kinematics and muscle activity in voluntary axial movement. J Electromyogr Kinesiol, 2003, 13 : 239-252. [Medline] [CrossRef] 